\title{
A new locus on 3p23-p25 for an autosomal-dominant limb-girdle muscular dystrophy, LGMD1H
}

\author{
Luigi Bisceglia ${ }^{1}$, Stefano Zoccolella ${ }^{2}$, Alessandra Torraco ${ }^{3,4}$, Maria Rosaria Piemontese $^{1}$, Rosa Dell'Aglio ${ }^{3}$, \\ Angela Amati ${ }^{5}$, Patrizia De Bonis ${ }^{1}$, Lucia Artuso ${ }^{3}$, Massimiliano Copetti ${ }^{6}$, Filippo Maria Santorelli ${ }^{4}$, \\ Luigi Serlenga $^{7}$, Leopoldo Zelante ${ }^{1}$, Enrico Bertini ${ }^{4}$ and Vittoria Petruzzella ${ }^{\star, 3,8}$
}

\begin{abstract}
Limb-girdle muscular dystrophies (LGMDs) are a genetically heterogeneous group of neuromuscular disorders with a selective or predominant involvement of shoulder and pelvic girdles. We clinically examined 19 members in a four-generation Italian family with autosomal-dominant LGMD. A total of 11 subjects were affected. Clinical findings showed variable expressivity in terms of age at onset and disease severity. Five subjects presented with a slowly progressive proximal muscle weakness, in both upper and lower limbs, with onset during the fourth-fifth decade of life, which fulfilled the consensus diagnostic criteria for LGMD. Earlier onset of the disease was observed in a group of patients presenting with muscle weakness and/or calf hypertrophy, and/or occasionally high CK and lactate serum levels. Two muscle biopsies showed morphological findings compatible with MD associated with subsarcolemmal accumulation of mitochondria and the presence of multiple mitochondrial DNA deletions. A genome-wide scan performed using microsatellite markers mapped the disease on chromosome 3p23-p25.1 locus in a 25-cM region between markers $D 3 S 1263$ and $D 3 S 3685$. The highest two-point LOD score was $3.26(\theta=0)$ at marker $D 3 S 1286$ and $D 3 S 3613$, whereas non-parametric analysis reached a $P$-value $=0.0004$. Four candidate genes within the refined region were analysed but did not reveal any mutations. Our findings further expand the clinical and genetic heterogeneity of LGMDs. European Journal of Human Genetics (2010) 18, 636-641; doi:10.1038/ejhg.2009.235; published online 13 January 2010
\end{abstract}

Keywords: autosomal-dominant limb-girdle muscular dystrophy; multiple mtDNA deletions; linkage analysis; chromosome 3

\section{INTRODUCTION}

The limb-girdle muscular dystrophies (LGMDs) are a heterogeneous group of hereditary neuromuscular disorders with predominant or selective weakness in proximal limb and axial muscles, having an estimated incidence of 1:100 000. The clinical phenotype is characterised by a great variability, ranging from early onset, with a severe and rapidly progressive clinical progression to milder forms, with a later onset and a slower progression. ${ }^{1}$ To date, seven autosomaldominant $(\mathrm{AD})$ and 12 autosomal-recessive (AR) forms of LGMD have been described, with $\mathrm{AD}$ families representing less than $10 \%$ of the whole group of LGMDs. Among the AD forms, LGMD1A caused by mutations in the TTID gene encoding myotilin on chromosome $5 \mathrm{q} 31$ is characterised by the presence of dysarthric speech - which probably relates to palatal weakness - in about $50 \%$ of the patients. ${ }^{2}$ LGMD1B, due to mutations in LMNA gene encoding lamin $\mathrm{A} / \mathrm{C}$ on chromosome 1q21, and LGMD1E, on 6q23, are also associated with heart conduction system abnormalities, including atrio-ventricular blocks, arrhythmia, and sudden death. ${ }^{3-5}$ Mutations in CAV3 gene, located on chromosome 3p25 (LGMD1D), encoding caveolin are associated with high creatine kinase (CK) levels with normal strength and distal myopathy, and usually occur in early childhood. ${ }^{6}$ Both ${\text { LGMD } 1 D^{7} \text { and LGMD1F }}^{8,9}$ loci map to chromosome $7 \mathrm{q}$ and do not present distinctive clinical features. The LGMD1G form, associated with progressive limitation of fingers and toes flexion, has been positionally cloned on chromosome $4 \mathrm{p} 21$ in a Brazilian Caucasian family. ${ }^{10}$ Fewer than ten AD-LGMD families show allelism with Bethlem myopathy mapped to chromosomes $2 \mathrm{q} 37$ and $21 \mathrm{q} 22.3$ and, less frequently, with facioscapulohumeral dystrophy (FSHD) mapped to 4q35 locus (www.neuro.wustl.edu/neuromuscular/musdist/ lg.html). Pedigrees not linked to any of the listed loci warrant further genetic heterogeneity.

We describe the clinical, pathological, and genetic features of a large Italian family with a previously unknown form of AD-LGMD characterised by slowly progressive clinical features and mapped to chromosome 3p23-p25 on whole genome scan.

\section{SUBJECTS AND METHODS}

Clinical and morphological studies

A total of 36 individuals (17 men and 19 women) spanning four generations in a pedigree from southern Italy were investigated. Figure 1 shows a partial pedigree of this kindred with, at least, two instances of a father-to-son transmission and refers to the branches directly examined in this study. According to established guideline criteria, ${ }^{11}$ index subjects were considered affected by LGMD when neurological examination revealed a typical pattern of muscle weakness affecting the upper and lower girdles. However, we recognised a second group of relatives with younger age at onset or milder clinical presentation, or both, and considered them as affected for genome-wide purposes. Muscle strength was appraised using the MRC (British Medical Research Council) Scale. Age at onset was determined by recording the age

${ }^{1}$ Department of evolutive age, Medical Genetics Unit, IRCCS-Ospedale Casa Sollievo della Sofferenza, San Giovanni Rotondo, Foggia, Italy; ${ }^{2}$ Clinic of Nervous System Diseases, Ospedali Riuniti di Foggia, Foggia, Italy; ${ }^{3}$ Department of Medical Biochemistry, Biology and Physics, University of Bari, Bari, Italy; ${ }^{4}$ Molecular Medicine, IRCCS-Ospedale 'Bambino Gesù', Rome, Italy; ${ }^{5}$ Department of Neurological and Psychiatric Sciences, University of Bari, Bari, Italy; ${ }^{6}$ Biostatistics Unit, IRCCS-Ospedale Casa Sollievo della Sofferenza, San Giovanni Rotondo, Foggia, Italy; ${ }^{7}$ Operative Unit of Neurology 'L. Bonomo' Hospital, Andria, Italy; ${ }^{8}$ Institute of Biomembranes and Bioenergetics, National Research Council, Bari, Italy ${ }^{*}$ Correspondence: Professor V Petruzzella, Department of Medical Biochemistry, Biology and Physics, University of Bari, Piazza Giulio Cesare 11, 00165 Bari, Italy. Tel: +39 080 5448529; Fax: +39 080 5448538; E-mail: v.petruzzella@biochem.uniba.it

Received 19 March 2009; revised 16 November 2009; accepted 9 December 2009; published online 13 January 2010 


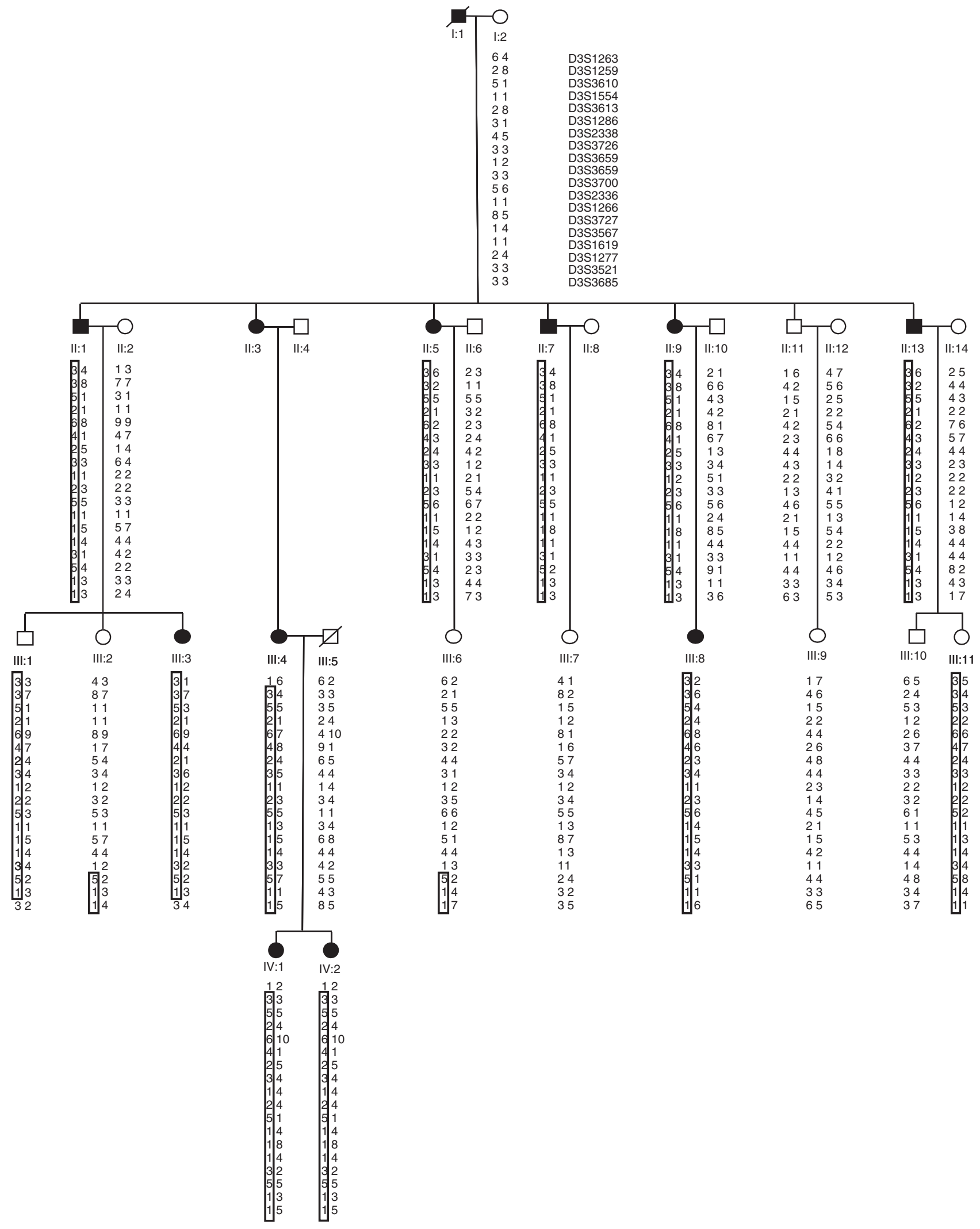

Figure 1 Pedigree of the family affected by the previously unknown AD LGMD form and haplotype analysis of microsatellite markers from chromosome 3p23-p25. The haplotype segregating with the disease is 'boxed'. Black filled symbols indicate affected subjects; clear symbols indicate the unaffected subjects. 
when muscle complains first became manifested. A total of 19 subjects (6 men and 13 women) were clinically assessed by two neurologists skilled in the evaluation of neuromuscular disorders. Clinical features of the remaining individuals were obtained by either family recall or consultation of available clinical notes. Routine blood tests were obtained to investigate metabolic causes of the disease. Serum CK and lactate levels were determined in all subjects. Electromyography (EMG) was performed in five patients. Four patients (II-1, II-5, II-9, and II-13) underwent a skeletal muscle biopsy. The specimens from the deltoid or vastus lateralis muscle were oriented, snap-frozen in liquid nitrogen-chilled isopentane, and cryostat-cut sections were stained using standard histochemical methods. Immunohistochemistry was performed using the following antibodies: anti-desmin and anti-vimentin (BioGenex Laboratories, San Ramon, CA, USA); anti-ubiquitin (Dako, Glostrup, Denmark); anti-Tau protein and anti- $\beta$-amyloid (Sigma, MO, USA); antisarcoglycans, anti-dystrophin, and anti- $\beta$-dystroglican (Becton-Dickinson, Franklin Lakes, NJ, USA); caveolin-3 (BD Transduction Laboratories, Lexington, KY, USA); and the amino terminal of utrophin (DRP2; Novocastra Laboratories, Newcastle upon Tyne, UK).

Having obtained the patients' informed consent, we purified genomic DNA from the blood of 19 members of the family, from six spouses, and from skeletal muscles of subjects II- 5 and II- 9 , using the standard phenol-chloroform procedure. Approximately $6 \mu \mathrm{g}$ of total DNA from muscle samples were cleaved with the endonuclease PvuII to linearise mtDNA molecules, electrophoresed on a $0.8 \%$ agarose gel, and blotted onto a nylon membrane (N+ Hybond, Amersham Biosciences, Little Chalfont, Buckinghamshire, UK). The entire mtDNA was PCR-amplified using four overlapping primer pairs (primers and PCR conditions can be made available on request), labelled with alpha- ${ }^{32} \mathrm{P}$-dATP by random priming (Random Primed DNA Labelling Kit, Roche Diagnostic, Mannheim, Germany), and used as a probe. Signal intensities were analysed with a Molecular Imager FX (Bio-Rad, Hercules, CA, USA).

\section{Linkage analysis}

A genome-wide scan was carried out by using the ABI PRISM Linkage Mapping Set LMS-MD10 (Applied Biosystems, Foster City, CA, USA), which includes 382 highly polymorphic microsatellite markers from 22 autosomes with an average intermarker distance of $10 \mathrm{cM}$. Additional, more densely spaced markers were used to refine chromosomal regions on $3 \mathrm{p}, 4 \mathrm{q}, 7 \mathrm{q}$, and $9 \mathrm{p}$ according to available genetic maps. Genotyping and fragments analysis was performed as previously described. ${ }^{12}$

Statistical analyses were carried out assuming an $\mathrm{AD}$ mode of inheritance with a disease allele frequency of 0.001 and assuming equal frequencies and recombination rates between men and women for all markers analysed. ${ }^{12}$ Physical and genetic distances between them were based on publicly available electronic databases from the University of California, Santa Cruz (www. genome.ucsc.edu) and the Marshfield genetic map (www.marshfieldclinic.org). Two age-dependent liability classes were considered for at-risk individuals: 0.80 in $<35$-year-old and 0.95 in $>35$-year-old subjects. In addition, because of the presence of two different groups of patients - the first made of definitively affected subjects and the second consisting of individuals with younger age at onset or milder clinical presentation, or both - we also conducted data analysis under a more stringent scenario, in which patients belonging to the second group were considered as having an 'unknown phenotype'. Data were simulated using SLINK ${ }^{13,14}$ to determine the power to detect linkage. Parametric twopoint linkage analysis was performed using MLINK from the Linkage Analysis Package II version 5.1, 1996. Non-parametric and parametric multipoint analyses were performed by means of the SimWalk2 programme version 2.83 that calculates the Maximum Location Score (directly comparable with multipoint LOD score) using Markov chain Monte Carlo (MCMC) and simulates annealing algorithm. ${ }^{15}$

\section{Sequencing of candidate genes}

The entire coding sequence of $C A V 3$ gene was amplified and directly sequenced in subjects II-1 and II-5 using previously published primer pairs and PCR conditions. ${ }^{6}$ The CMYA1, CAPN7, and MGC15763 genes were screened for mutations in the same individuals. The PCR primers were designed using the Primer 3 software $^{16}$ (sequences can be made available on request). All PCR fragments were agarose gel-purified and directly sequenced on both strands using the Big Dye Terminator chemistry on an ABI 3100 DNA sequencer (Applied Biosystems).

\section{RESULTS}

Table 1 summarises clinical, morphological, and laboratory data in the examined family members. The ID numbers refer to the pedigree represented in Figure 1. A subset of severely affected members developed a slowly progressive proximal muscle weakness in both upper and lower limbs; among these, five subjects (II-1, II-3, II-5, II-9, and II-13) were considered as affected as they fulfilled the criteria for the diagnosis of LGMD. ${ }^{11}$ We also identified a mildly affected group of patients (II:7, III:3, III:4, III:8, IV:1, and IV:2) who had calf hypertrophy, and occasional increase in CK and lactate levels. These patients were considered as possibly affected, though to a lesser extent.

On clinical grounds, onset of symptoms started around the fifth decade of life in five cases with a slowly progressive muscle weakness (median MRC score=4), initially in the lower limbs; hypotrophy of both upper and lower limb-girdles muscles; reduced deep tendon reflexes; and calf hypertrophy. In one case (I-1), age of onset and clinical phenotype were established by family recall and evaluation of medical records, but we could not examine him as he had died at the age of 77 years because of an ischaemic heart attack. On average, serum CK levels were elevated (ranging from 997-2300 U/1; normal $<230 \mathrm{U} / \mathrm{l})$, whereas serum lactate levels were normal. One patient (case II-13) had been defined healthy at age 39 years when examined elsewhere, though he complained of unspecified muscle fatigability, and occasional increase in serum CK. A four-year follow-up examination revealed mild weakness in the lower limb-girdle muscles (MRC 4-5) and elevated CK levels (1600 U/l). The EMG analysis showed a decreased duration of motor unit potentials, without the evidence of denervation in four limb muscles, in two cases (II-1; II-5). None of the patients presented ultrasound evidence of abnormal heart muscle function. A second, less severely affected group of individuals in this family had a relatively younger age at examination (median age $=28$ years), and displayed calf hypertrophy but no significant muscle weakness. Eight individuals (median age $=22$ years) were clinically healthy, did not complain of muscular symptoms, and had CK values in the normal range.

Histochemical analysis performed on muscle biopsy samples from patients II-1, II-5, II-9, and II-13 showed abnormal fibre size and shape variation, and increased presence of endo- and perimysial connective tissues on hematoxylin-eosin staining. Central nuclei were occasionally present. Ragged red fibres (RRFs) were observed in patient II-1 and subsarcolemmal accumulation of mitochondria on succinate dehydrogenase (SDH) staining, as well as fibres negative for cytochrome $c$ oxidase staining were evidenced in patients II-5 and II-9. Immunohistochemical stainings for dystrophin, sarcoglycan, caveolin, and $\delta$-sarcoglycan were normal. In addition to the wild-type mtDNA, Southern blotting in skeletal muscle biopsy samples from patients II-5 and II-9 showed multiple bands of lower size corresponding to deleted mtDNA molecules (Figure 2).

Following the initial $10-\mathrm{cM}$ genome-wide screening, multipoint analysis showed four chromosomal regions (3p23, 4q13.2, 7qter, and 9 p13.3) with a maximum location score $>2$. Using more densely spaced markers within each identified region, we increased the resolution map to $\sim 1.5 \mathrm{cM}$, and conclusively excluded linkage to the $4 \mathrm{q}$ and $7 \mathrm{q}$ regions. A maximum location score of 2.36 was obtained for chromosome 9p but the highest value of 3.236 was reached at marker D3S1286, located on chromosome 3p23-p25. 
Table 1 Principal clinical and laboratory data in family members

\begin{tabular}{|c|c|c|c|c|c|c|c|c|c|c|}
\hline & Patient & $\begin{array}{c}\text { Age at } \\
\text { diagnosis }\end{array}$ & $\begin{array}{l}\text { Age at } \\
\text { onset }\end{array}$ & Sex & Biopsy $^{\mathrm{a}}$ & $E M G$ & $\begin{array}{c}\text { CK level } \\
(U / I)\end{array}$ & $\begin{array}{l}\text { Lactate level } \\
(\mathrm{mg} / 100 \mathrm{ml})\end{array}$ & $\begin{array}{l}\text { Neuromuscular } \\
\text { examination }\end{array}$ & Other symptoms \\
\hline \multirow[t]{7}{*}{ Severely affected } & $\mathrm{I}: 1^{\mathrm{b}}$ & Dead at 77 years & - & M & NA & NA & NA & NA & Calf hypertrophy & Headache; heart problems \\
\hline & II:1 & 60 & 50 & M & ++ & Myopathic pattern & 2295 & 13.2 & $\begin{array}{l}\text { Muscle weakness; } \\
\text { calf hypertrophy }\end{array}$ & Dyspnea; scoliosis \\
\hline & $11: 3^{c}$ & 59 & 49 & $\mathrm{~F}$ & NA & Myopathic pattern & 1551 & 11.0 & Muscle weakness & \\
\hline & $11: 5$ & 57 & 50 & $\mathrm{~F}$ & ++ & Myopathic pattern & 997 & 8.7 & Muscle weakness & \\
\hline & $11: 9$ & 49 & 45 & $\mathrm{~F}$ & ++ & Myopathic pattern & 2139 & 6 & $\begin{array}{l}\text { Muscle weakness; } \\
\text { calf hypertrophy }\end{array}$ & Scoliosis \\
\hline & $11: 13$ & 42 & 39 & M & ++ & Normal & 1600 & 10.7 & Muscle weakness & \\
\hline & III:3 & 24 & 20 & $\mathrm{~F}$ & NA & NA & 2300 & 11.9 & Muscle weakness & \\
\hline \multirow[t]{5}{*}{ Mildly affected } & II:7 & 50 & 46 & M & NA & NA & 153 & 20.5 & Calf hypertrophy & \\
\hline & III:4 & 37 & 34 & $\mathrm{~F}$ & NA & NA & 160 & 18.2 & Calf hypertrophy & Headache \\
\hline & III:8 & 22 & 22 & $\mathrm{~F}$ & NA & NA & 155 & 38.7 & Calf hypertrophy & \\
\hline & IV:1 & 17 & 17 & $\mathrm{~F}$ & NA & NA & 67 & 11.6 & Calf hypertrophy & \\
\hline & $\mathrm{IV}: 2$ & 16 & 16 & $\mathrm{~F}$ & NA & NA & 98 & 11.3 & Calf hypertrophy & \\
\hline \multirow[t]{8}{*}{ Unaffected } & $\|: 11$ & 45 & - & M & NA & NA & 62 & 8.2 & Normal & \\
\hline & $\| I I: 1$ & 30 & - & M & NA & NA & 185 & 10.9 & Normal & \\
\hline & III:2 & 27 & - & $\mathrm{F}$ & NA & NA & 110 & 12.3 & Normal & \\
\hline & III:6 & 10 & - & $\mathrm{F}$ & NA & NA & 97 & 11.1 & Normal & \\
\hline & III:7 & 10 & - & $\mathrm{F}$ & NA & NA & 183 & 9.9 & Normal & \\
\hline & III:9 & 24 & - & $\mathrm{F}$ & NA & NA & 78 & 11.7 & Normal & \\
\hline & III:10 & 17 & - & M & NA & NA & 162 & 8.1 & Normal & \\
\hline & III:11 & 14 & - & $\mathrm{F}$ & NA & NA & 130 & 11.3 & Normal & Recurrent migraine without aura \\
\hline
\end{tabular}

++ , skeletal muscle biopsy showing dystrophic pattern; NA, not available.

aDescription of bioptical findings in the text.

bClinical information based on anamnestic recall.

cConsultation of clinical notes.

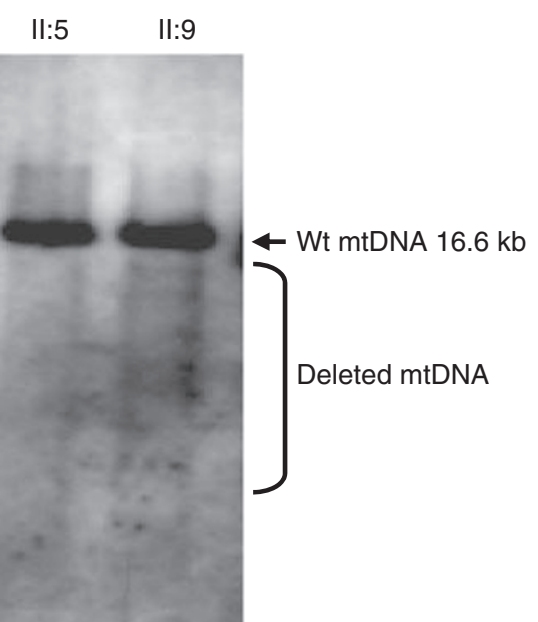

Figure 2 Southern blot performed on DNA extracted from skeletal muscle biopsy samples.

A value $>3.0$ was observed in the $\sim 16 \mathrm{cM}$ interval between markers D3S3610 and D3S1266 (Figure 3). Two-point analysis showed an MLS value $=3.23(\theta=0.0)$ at markers D3S3613 and D3S1286 (Table 2). Nonparametric multipoint analysis supported this conclusion at both markers, yielding a STAT D score of $2.26(P=0.0004)$ and a STAT E score of $15.4358(P=0.0004)$, in which 'statistic D' is the extent of allele sharing among all affected pairs as measured by their IBD kinship coefficient, ${ }^{15}$ and 'statistic E' is the NPL_all statistic as implemented in GeneHunter ${ }^{17}$ (Table 2). When we adopted a more conservative analysis, which considers 'unknown' cases as belonging to the group of mildly affected patients, the highest value of LOD score (MLS $>1.50$ ) was reached in the interval between markers D3S3610 and D3S3726. Taken together, our results made chromosome 3 p23-25p the most likely region where the new LGMD1H locus could be potentially located.

Haplotype reconstruction confirmed the results obtained from linkage analysis data (Figure 1). The telomeric boundary of the candidate interval was defined by an obligate recombination event observed between D3S1263 and D3S1259 in subject III-4, whereas the centromeric flanking marker is defined by another recombination event between markers D3S3521 and D3S3685 in subject III-3. All affected individuals shared the same disease haplotype. Two healthy individuals (III-2 and III-6) shared part of the same haplotype between markers D3S1619 and D3S1277, thus further refining the LGMD1H locus to a 25-cM region.

Two presently unaffected individuals (III-1 and III-11) shared the disease haplotypes. Subject III-1 is a 30 -year-old man with normal serum CK levels. Subject III-11 is a 14-year-old girl with a history of recurrent migraine who has never had muscle-related complaints or high CK levels. However, she is younger than the median age at onset of her affected relatives. Neurological examinations in both individuals were normal.

The region refined by linkage analysis contains more than 150 genes. Four possible candidates were prioritised for mutational screening because of their function or position: CAV3, CAPN7, MGC15763, and CMYA1. Although located well outside the interval refined in linkage with this family, the caveolin-3 gene (CAV3) was analysed to firmly exclude allelism with LGMD1C. The CAPN7 gene encodes an 
814-residue protein that contains a calpain-like cysteine protease domain and a C-terminal $\mathrm{PBH}$ domain. Expressed mostly in the pancreas and only moderately in the skeletal muscle and liver, ${ }^{18}$ CAPN7 shares sequence homologies with CAPN3, the LGMD2A causative gene. The MGC15763 gene encodes for a 312-amino acid-long protein that is predicted to be exported to mitochondria (MitoProt II $1.0 \alpha 4$ probability is 0.8599 ; predicted cleavage site at amino acid 44). According to primary structural analysis, the gene product has a NADH-binding domain but no strong homology with any known protein. The CMYA1 gene encodes the human orthologue of $\operatorname{mXin} \alpha$, a protein believed to have a regulatory role in cardiac morphogenesis, differentiation, and to be localised to the adherence junctions of intercalated discs. ${ }^{19}$ The entire coding regions of CAV3,

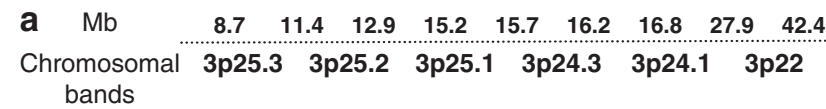

b

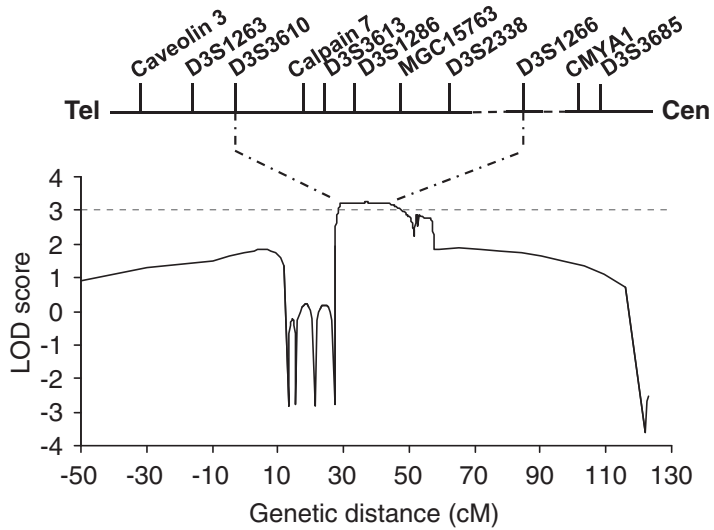

Figure 3 Diagrammatic representation of the region 3p22-p25.3. (a) Schematic map of the short arm of the chromosome 3 showing markers and genes of interest located between 11 and $42 \mathrm{Mb}$. (b) Multipoint LOD scores between the disease and markers on chromosome $3 p$.
CAPN7, MGC15763 and CMYA1 and their flanking intronic sequences were analysed, but no pathogenic mutations were found.

\section{DISCUSSION}

We identified a large pedigree from southern Italy segregating a previously unknown $\mathrm{AD}$ form of muscular dystrophy, which fulfilled the diagnostic criteria for LGMDs. ${ }^{11,20}$ Variable expressivity was observed in term of age at onset and muscular symptoms. With few exceptions, the adult patients showed the 'canonical' clinical features of LGMD, characterised by slowly progressive proximal muscle weakness in upper and lower limbs, with onset during the fifth decade of life and a relatively benign course. Neither involvement of ocularfacial-bulbar muscles, nor cardiac muscle involvement, nor early joint contractures were observed. A single individual (I:1) had a likely hypertensive cardiomyopathy, whereas echocardiography performed in three subjects affected by LGMD (II:1, II:3, II:13) was normal. An earlier onset of the disease was found in a group of patients complaining muscle weakness or calf hypertrophy and occasional increase in CK and lactate levels. On the basis of the clinical history of five adult members, this juvenile group is likely to develop the typical pattern of LGMD later in life. Two unaffected subjects (III:1 and III:11) carried the disease haplotype, suggesting an incomplete penetrance for the disease. However, the follow-up for the subject III:11 remains unpredictable, as she is younger than the mean age of second-generation relatives.

Consistently, immunohistochemistry and linkage analysis excluded any other known dominant form of LGMD. In keeping with the current classification of inherited LGMDs, we propose to define this form of muscular dystrophy as LGMD1H.

Evidence of histochemical features, suggestive of a defect in oxidative phosphorylation and multiple mtDNA deletions in skeletal muscle from two patients, seemed to suggest a mitochondrial dysfunction. A similar feature was observed in one patient from the only LGMD1F family so far reported. ${ }^{8}$ Multiple mtDNA rearrangements usually result from mutations in the genes involved in mtDNA replication and maintenance. ${ }^{21}$ At least seven genes have been identified in this subset of mitochondrial disorders (for review, see Zeviani et $a^{22}$ ), but

Table 2 Two-point LOD score and multipoint non-parametric LOD scores between LGMD and microsatellite markers on chromosome 3p23-p25

\begin{tabular}{|c|c|c|c|c|c|c|c|c|c|c|c|c|c|}
\hline \multirow[b]{2}{*}{ Markers } & \multicolumn{2}{|c|}{ Position } & \multicolumn{7}{|c|}{ Two-point $L O D$ score at $\theta_{(m=f)}=$} & \multicolumn{4}{|c|}{ Multipoint NPL } \\
\hline & $c M$ & $M b$ & 0.0 & 0.01 & 0.05 & 0.1 & 0.2 & 0.3 & 0.4 & STAT D & P-value & STAT E & P-value \\
\hline D3S1263 & 36.10 & 11.492 & -2.52 & 0.90 & 1.47 & 1.57 & 1.39 & 0.97 & 0.44 & 1.3998 & 0.2250 & 0.1383 & 0.1527 \\
\hline D3S1259 & 36.65 & 12.073 & 2.32 & 2.30 & 2.21 & 2.06 & 1.66 & 1.14 & 0.53 & 1.8495 & 0.1087 & 8.1269 & 0.0746 \\
\hline D3S3610 & 37.20 & 12.980 & 0.66 & 0.66 & 0.63 & 0.59 & 0.46 & 0.31 & 0.15 & 2.2600 & 0.0010 & 15.4095 & 0.0008 \\
\hline D3S1554 & 39.52 & 14.342 & 0.88 & 0.90 & 0.92 & 0.86 & 0.71 & 0.49 & 0.24 & 2.2591 & 0.0005 & 15.3722 & 0.0004 \\
\hline D3S3613 & 41.56 & 15.336 & 3.23 & 3.19 & 3.05 & 2.83 & 2.27 & 1.58 & 0.76 & 2.2614 & 0.0005 & 15.4394 & 0.0005 \\
\hline D3S1286 & 41.56 & 15.794 & 3.23 & 3.19 & 3.05 & 2.83 & 2.27 & 1.58 & 0.76 & 2.2615 & 0.0004 & 15.4396 & 0.0004 \\
\hline D3S2338 & 42.10 & 16.824 & 2.75 & 2.72 & 2.57 & 2.35 & 1.81 & 1.16 & 0.48 & 2.2615 & 0.0005 & 15.4397 & 0.0004 \\
\hline D3S3726 & 42.64 & 19.509 & 2.21 & 2.18 & 2.02 & 1.81 & 1.37 & 0.88 & 0.36 & 2.2611 & 0.0005 & 15.4268 & 0.0004 \\
\hline D3S3659 & 47.44 & 22.913 & 1.23 & 1.21 & 1.13 & 1.01 & 0.73 & 0.42 & 0.12 & 2.2610 & 0.0002 & 15.4261 & 0.0002 \\
\hline D3S3700 & 48.09 & 23.885 & 2.75 & 2.72 & 2.57 & 2.35 & 1.80 & 1.15 & 0.46 & 2.2624 & 0.0002 & 15.4443 & 0.0002 \\
\hline D3S2336 & 49.18 & 24.889 & 1.75 & 1.73 & 1.63 & 1.44 & 1.15 & 0.74 & 0.29 & 2.2614 & 0.0004 & 15.4163 & 0.0004 \\
\hline D3S1266 & 52.60 & 27.932 & 2.21 & 2.18 & 2.02 & 1.81 & 1.37 & 0.88 & 0.36 & 2.2608 & 0.0007 & 15.4012 & 0.0005 \\
\hline D3S3727 & 56.07 & 30.652 & 1.21 & 1.24 & 1.28 & 1.26 & 1.06 & 0.74 & 0.37 & 2.2622 & 0.0008 & 15.4436 & 0.0007 \\
\hline D3S3567 & 56.69 & 30.713 & 1.20 & 1.18 & 1.10 & 0.97 & 0.66 & 0.32 & 0.07 & 2.2622 & 0.0007 & 15.4436 & 0.0007 \\
\hline D3S1619 & 60.98 & 34.087 & 2.62 & 2.60 & 2.49 & 2.32 & 1.87 & 1.29 & 0.60 & 2.3114 & 0.0005 & 15.7125 & 0.0004 \\
\hline D3S1277 & 61.52 & 34.627 & 1.83 & 1.84 & 1.84 & 1.79 & 1.54 & 1.11 & 0.53 & 2.3241 & 0.0004 & 15.7821 & 0.0003 \\
\hline D3S3521 & 63.12 & 38.830 & 1.23 & 1.24 & 1.29 & 1.28 & 1.13 & 0.82 & 0.37 & 2.3136 & 0.0005 & 15.3653 & 0.0004 \\
\hline D3S3685 & 67.94 & 42.429 & -7.75 & -1.46 & -0.10 & 0.40 & 0.70 & 0.61 & 0.29 & 1.7626 & 0.0321 & 0.6551 & 0.0331 \\
\hline
\end{tabular}


none locate in the 3p23-p25 interval defined in our LGMD1H family. Low levels of multiple mtDNA deletions can also be a secondary event, such as it happens in sporadic and familial inclusion body myosites ${ }^{23}$ and in the ageing processes. ${ }^{24}$ In our patients, mtDNA deletions are likely to be considered as by-products of mitochondrial dysfunction of still unknown significance.

Whole-genome scan for the LGMD1H locus showed data compatible with linkage to a 31-cM region on chromosome 3p23-p25. Haplotype reconstruction allowed the refinement of the candidate region to 25-cM between markers D3S1263 and D3S1277. In this interval, in silico analyses revealed the presence of more than 150 genes (www.ensembl.org). However, no evident disease-causative gene emerged, and two of the potential candidates (CAPN7 and MGC15763) were ruled out by direct sequencing. The function of the human CAPN7 gene product is presently unknown, but the mouse orthologue is thought to be a calcium-independent enzyme with protease activity. The human protein seems to have a nuclear localisation and is the most divergent element in the calpain team, sharing only $26-35 \%$ of its sequence with the other members of this family. ${ }^{18}$ The MGC15763 gene encodes the hypothetical protein $\mathrm{BC} 008322$ that contains a region of NADH-cytochrome $b$ reductase involved in coenzyme transport and metabolism, energy production and conversion, and it is predicted to have a mitochondrial targeting.

In summary, we have presented evidence for a previously unknown form of AD-LGMD, mapping on chromosome 3p23-p25.1, characterised by a slow progression of proximal muscle weakness in both upper and lower limbs. The transmission model of the disease is consistent with an $\mathrm{AD}$ pattern of inheritance with variable expressivity and incomplete penetrance. In fact, symptoms vary in terms of age at onset and clinical severity. The identification of additional LGMD1H families or further refinement of the genetic interval in our kindred, or both is needed before considering screening for additional candidate genes.

\section{CONFLICT OF INTEREST}

The authors declare no conflict of interest.

\section{ACKNOWLEDGEMENTS}

This study was supported in part by grants from the Italian Ministry of Health 'Ricerca Corrente 2009-2011'; MIUR-PRIN 'Molecular mechanisms, physiology and pathology of membrane bioenergetics system', Project No. 2005052128_001; and Research Foundation Cassa di Risparmio di Puglia.

1 Beckmann JS, Brown RH, Muntoni F, Urtizberea A, Bonnemann C, Bushby KMD: 66th/67th ENMC sponsored international workshop: the limb-girdle muscular dystrophies, 26-28 March 1999, Narden, the Nederlands. Neuromuscular Disord 1999; 9: 436-445.

2 Hauser MA, Horrigan SK, Salmikangas $P$ et al: Myotilin is mutated in limb girdle muscular dystrophy 1A. Hum Mol Genet 2000; 9: 2141-2147.

3 van der Kooy AJ, van Meegen M, Ledderhof TM et al: Genetic localization of a newly recognized autosomal dominant limb-girdle muscular dystrophy with cardiac involvement (LGMD1B) to chromosome 1q 11-21. Am J Hum Genet 1997; 60. 891-895.

4 Muchir A, Bonne G, van der Kooi AJ et al: Identification of mutation in the gene encoding lamins $A / C$ in autosomal dominant limb-girdle muscular dystrophy with atrioventricular conduction disturbances (LGMD1B). Hum Mol Genet 2000; 9: 1453-1459.

5 Messina DM, Speer MC, Pericak-Vance MA, McNally EM: Linkage of familial dilated cardiomyopathy with conduction defect and muscular dystrophy to chromosome 6q23. Am J Hum Genet 1997; 61: 909-917.

6 Minetti C, Sotgia F, Bruno C et al: Mutations in the caveolin-3 gene cause autsomal dominant limb-girdle muscular dystrophy. Nat Genet 1998; 18: 365-368.

7 Speer MC, Vance JM, Grubber JM et al: Identification of a new autosomal dominant limb-girdle muscular dystrophy locus on chromosome 7q. Am J Hum Genet 1999; 64: 556-562.

8 Gamez J, Navarro C, Andreu AL et al: Autosomal dominant limb-girdle dystrophy: a large kindred with evidence of anticipation. Neurology 2001; 56: 450-454.

9 Palenzuela L, Andreu AL, Gamez J et al: A novel autosomal dominant limb-girdle muscular dystrophy (LGMD1F) maps to 7q32.1-32.2. Neurology 2003; 61: 404-406.

10 Starling A, Kok F, Passos-Bueno MR, Vainzof M, Zatz M: A new form of autosomal dominant limb-girdle muscular dystrophy (LGMD1G) with progressive fingers and toes flexion limitation maps to chromosome 4p21. Eur J Hum Genet 2004; 12: 1033-1040.

11 Bushby KMD: Limb girdle muscular dystrophy; in Emery AEH (ed).: Diagnostic Criteria for Neuromuscular Disorders. London, UK: Royal Society of Medicine Press, 1997. pp 17-22.

12 Bisceglia L, De Bonis P, Pizzicoli C et al: Linkage analysis in keratoconus: replication of locus 5q21.2 and identification of other suggestive loci. Invest Ophthalmol Vis Sci 2009; 50: 1081-1086.

$13 \mathrm{Ott} \mathrm{J}$ : Computer-simulation methods in human linkage analysis. Proc Natl Acad Sci USA 1989; 86: 4175-4178.

14 Weeks DE, Ott J, Lathrop GM: SLINK: a general simulation program for linkage analysis. Am J Hum Genet 1990; 47: A204.

15 Sobel E, Lange K: Descent graphs in pedigree analysis: applications to haplotyping, location scores, and marker sharing statistics. Am J Hum Genet 1996; 58: 1323-1337.

16 Rozen S, Skaletsky HJ: Primer3 on the WWW for general users and for biologist programmers; in Krawetz S, Misener S (eds): Bioinformatics Methods and Protocols. Totowa, NJ: Humana Press, 2000. pp 365-386.

17 Whittemore AS, Halpern J: Probability of gene identity by descent: computation and applications. Biometrics 1994; 50: 109-117.

18 Futai E, Kubo T, Sorimachi H, Suzuki K, Maeda T: Molecular cloning of PalBH, a mammalian homologue of the Aspergillus atypical calpain PalB. Biochim Biophys Acta 2001; 1517: 316-319.

19 Jung-Ching Lin J, Gustafson-Wagner EA, Sinn HW et al: Structure, expression, and function of a novel intercalated disc protein, Xin. J Med Sci 2005; 25: 215-222.

20 Guglieri M, Straub V, Bushby K, Lochmüller H: Limb-girdle muscular dystrophies. Curr Opin Neurol 2008; 21: 576-584.

21 Di Mauro S, Schon EA: Mitochondrial respiratory-chain diseases. N Engl J Med 2003; 348: 2656-2668.

22 Zeviani M, Spinazzola A, Carelli V: Nuclear genes in mitochondrial disorders. Curr Opin Genet Dev 2003; 13: 262-270.

23 Jansson $M$, Darin N, Kyllerman M, Martinsson T, Wahlström J, Oldfors A: Multiple mitochondrial DNA deletions in hereditary inclusion body myopathy. Acta Neuropathol 2000; 100: 23-28.

24 Fayet G, Jansson M, Sternbergd D et al: Ageing muscle: clonal expansions of mitochondrial DNA point mutations and deletions cause focal impairment of mitochondrial function. Neuromuscular Disord 2002; 12: 484-493. 